\title{
Biodiversity crisis or sixth mass extinction?
}

Does the current anthropogenic biodiversity crisis really qualify as a mass extinction?

\section{Valentí Rull}

Botantic Institute of Barcelona, Pg. del Migdia s/n, 08038 Barcelona, Spain

Email: vrull@csic.es

Five centuries ago, maritime technology experienced significant improvements that allowed European peoples to travel to almost any corner of the planet, especially to oceanic islands. This resulted in large and irreversible ecological modifications of the visited lands, including major landscape disturbances and the extinction of many native species. Since then, anthropogenic extinction has not stopped but, on the contrary, has been magnified worldwide due to generalized overexploitation of natural resources, habitat destruction/fragmentation and other destructive alterations, which have led to an outstanding biodiversity depletion usually referred to as the current anthropogenic biodiversity crisis. It has also been anticipated that ongoing global change will contribute to exacerbating anthropogenic extinction in the near future. A couple of decades ago, some scientists suggested that the ongoing anthropogenic biodiversity crisis could have the magnitude of a mass extinction similar to those that occurred in past geologic times, especially the five major mass extinctions, and coined the term "sixth extinction" (Leakey \& Levin, 1995). This term and its equivalent "sixth mass extinction" have become popular in both scientific and nonscientific environments and are frequently used by scientists, environmentalists, popular writers and journalists, among others.

Many people uncritically accept the reality of the sixth mass extinction, whereas others believe that this is an unrealistic exaggeration promoted by environmental alarmists (Lomborg, 2001). In between, part of society does not know what to believe due to the lack of more specific information. Therefore, whether the current biodiversity crisis qualifies as a mass extinction remains unclear in many social sectors, including to nonspecialist scholars. This essay is an attempt to clarify the issue on the basis of the available scientific evidence. The first part discloses the number and the main features of anthropogenic extinctions that have occurred since 1500 . Then, these numbers are compared with those of the five mass extinctions that occurred during the Phanerozoic (the last 540 million years or $\mathrm{Ma}$ ) to determine whether the magnitudes are similar. First of all, however, it seems necessary to stress that extinction, rather than something intrinsically bad, is a common phenomenon that is essential for biological diversification, as diversification rates result from the balance between the rates of species origination (speciation) and disappearance (extinction).

\section{Background extinction}

In the context of this discussion, it is important to distinguish between background extinction and episodic extinction. Background extinction is a usual process in the evolutionary play, which depends on ecological and biogeographical factors (competition, predation, diseases, habitat loss, climatic changes, dispersal, range shifts, etc.), under the rule of natural selection. This type of extinction continuously balances speciation in the generation of net spatiotemporal biodiversity patterns. A common measure of extinction rates is the number of extinctions per million species-years (E/MSY), which is inversely proportional to the duration of 
species within a given group (Pimm et al., 1995). For example, it has been estimated that the average duration of mammalian species is $10^{6}$ years and, therefore, their background extinction is $1 \mathrm{E} / \mathrm{MSY}$, meaning one extinction per million species per year or one extinction per $10^{4}$ species per century. In the case of marine invertebrates, whose average species duration is $10^{7}$ years, background extinction is $0.1 \mathrm{E} / \mathrm{MSY}$. Species of most animal groups have intermediate durations (Lawton \& May, 1995); hence, background extinction rates fluctuate between 0.1 and $1 \mathrm{E} / \mathrm{MSY}$ for most animal groups. Episodic extinction occurs unexpectedly when a major force causes an abrupt extinction burst significantly exceeding background extinction, which can be measured by E/MSY values. For example, if an extinction burst reduces the average duration of mammalian species to $10^{5}$ years, then $\mathrm{E} / \mathrm{MSY}$ values increase to $10\left(10^{6} / 10^{5}\right)$, which highly exceeds background extinction rates. It should be stressed, however, that not all episodic extinction events qualify as mass extinctions, as we will see later.

\section{Anthropogenic extinctions}

Coming back to the central issue of this essay, the most reliable source with which to assess the magnitude and patterns of current anthropogenic extinctions is the IUCN (International Union for Conservation of Nature) Red List of threatened species

(https://www.iucnredlist.org/; retrieved 16 October 2021). Currently, this list contains nearly 140,000 well-documented species, of which 900 have already gone extinct (EX) since the year 1500 and almost 80 are extinct in the wild (EW), though preserved in cultivation. Species that are still living but are threatened with extinction are grouped into three categories: critically endangered (CR), endangered and vulnerable (VU). The focus here is on the 900 extinct species, most of which (86\%) are animals and the remaining $14 \%$ are plants (mostly flowering plants, except for a few fern and moss species). Among animals, one-third of the extinct species are mollusks, followed by birds, mammals, fishes, insects, amphibians and reptiles. Crustaceans, arachnids, worms and myriapods account for around or below $1 \%$ (Fig. 1). Most of these extinctions occurred in the Pacific islands and the Americas (30\% each) and tropical Africa (20\%); less affected areas include Eurasia, Australia and the Indomalayan region (all of them with values below $10 \%)$. The causes for the extinction of these species were mostly anthropogenic, including overhunting, replacement by introduced species, deforestation, habitat destruction, increased land use and/or introduction of alien pathogens. Size and connectivity constraints make oceanic islands especially sensitive to these anthropogenic disturbances (Rull, 2021).

The above numbers may be greater if we consider so-called dark extinction, that is, the extinction of species before they are discovered and named. Although difficult to quantify globally, a recent study estimated that dark extinction in birds could have increased their IUCN extinction numbers by $150 \%$ (Boehm \& Cronk, 2021). However, no similar studies exist for other groups. This number may still increase by considering prehistoric extinctions, which are only detectable in the fossil record. However, the incompleteness of the fossil record and the difficulties in attributing extinctions to human causes hinder reliable estimates. This is, for example, the case for global megafaunal extinctions that occurred between about 50 and 10 kiloyears before present (kyr BP), whose cause, climatic and/or human, remains unresolved (Koch \& Barnosky, 2006). More reliable estimates exist for anthropogenic faunal extinctions predating historical records. The most complete study in this sense is that of oceanic Pacific islands where based on subfossil bone records from archaeological sites, more than 2000 bird species would have been extinct because of anthropogenic causes before European contact (Steadman 1995). Once more, these estimates are based on birds and are of regional rather than global scope. Therefore, the IUCN Red List is still the reference for the number of extinctions during the last 500 years. 
The Encyclopedia of Life (EOL) has actually documented 1.9 million living species on Earth and estimates that there are a total of 8.7 million species (https://www.eol.org/; retrieved 16 October 2021). Then, the 900 IUCN-documented extinct species represent $0.5 \%$ of the known species and $0.01 \%$ of Earth's estimated total biodiversity. In temporal terms, the IUCN extinctions represent an average rate of 180 extinctions per century (1.8 extinctions per year). How does this anthropogenic biodiversity depletion compare to the five major mass extinctions that occurred in past geological times?

\section{Comparison with past mass extinctions}

The Big Five mass extinctions have been defined on the basis of the fossil record of marine animals, which has been considered to be representative of global biodiversity trends, at least in relative terms. In this way, a number of episodic extinctions - i.e., above the background extinction levels - have been identified, of which five have been defined as mass extinctions (Fig. 2). These mass extinctions have been attributed to endogenous and exogenous biospheric causes, notably meteorite impacts; global shifts in climate and/or atmospheric/oceanic biogeochemistry; recurrent marine transgressions coupled or not with eutrophication and deep-water anoxic events; and generalized increases in volcanism, uplift and weathering episodes, among others. By convention, the condition for an episodic extinction burst to be considered a mass extinction is that $75 \%$ or more of the living species go extinct in a couple million years or less, sometimes much less (Barnosky et al., 2011).

According to the above estimates, the current anthropogenic biodiversity crisis initiated in 1500 does not qualify as a mass extinction comparable to the Big Five, as the number of species that went extinct during the last 500 years ranges between $0.5 \%$ and $0.01 \%$ of the documented and the estimated living species, respectively. However, if we consider the average rate of 180 extinctions per century, $75 \%$ of the total known species (1.4 million species) would be extinct in $800 \mathrm{kyr}$, and the same percentage of the total estimated species (6.5 million species) would be extinct in 3.6 million years. Therefore, according to the above paleontological definition, the rates of the current biodiversity crisis are within the range of magnitudes of a mass extinction. If we were able to add human-driven dark and prehistoric extinctions, of which we have no reliable estimates, extinction rates would be even greater and the time for attaining the $75 \%$ extinction boundary would be significantly shorter. But the situation might be even worse, as the IUCN extinction records seem to be underestimated for the period before 1800 .

Although data on extinction time are unavailable for about one-third of the 900 IUCNdocumented extinctions, $90 \%$ of the documented species went extinct in the last two centuries, which could be attributed to a "taxonomy effect". Indeed, modern taxonomy has its roots in the binomial system of Carl Linnaeus, which was generally adopted in the early $19^{\text {th }}$ century. The period between 1500 and 1800 has been called the pretaxonomic period, as most current species were described after 1800 (Boehm \& Cronk, 2021). Therefore, extinction rates before the $19^{\text {th }}$ century are difficult to ascertain. It is also worth noting that field collections underwent an overwhelming increase in 1900. Therefore, the most robust and welldocumented extinction rates are those of the $20^{\text {th }}$ century, with an average of 3.6 species per year, which double the average rates for the 1500-1800 period. If these rates are maintained, a $75 \%$ biodiversity loss would be attained in "only" 400 kyr for the known species and 1.8 million years for Earth's estimated biodiversity. This falls within the range of a mass extinction, as defined paleontologically. According to these figures, we might actually be at the beginning of the sixth mass extinction, this time caused by humans (Leakey \& Lewin, 1992). 
It is interesting to compare the extinction values documented by the IUCN Red List with the estimated background extinction. This comparison cannot be general due to the lack of reliable background E/MSY values for some groups, but in the case of vertebrates (mammals, birds, fishes, reptiles and amphibians), for which average background extinction has been estimated to be $1.8 \mathrm{E} / \mathrm{MSY}$, nine extinctions would be expected for the $20^{\text {th }}$ century (Ceballos et al., 2015). Instead, 390 vertebrate species have gone extinct since 1900, which is more than 40 times the background extinction for this group of animals.

\section{Future projections}

Given the present state of knowledge, it is not possible to anticipate whether our species will continue to behave in the same way and at the same rates for a couple million years, or even if we will continue thriving on Earth (we have been here for barely $200 \mathrm{kyr}$ ) or we will go extinct. In spite of this, future projections are based on the maintenance of our present-day standards. For example, the Intergovernmental Science-Policy Platform on Biodiversity and Ecosystem Services (IPBES, 2019) gathered nearly 15,000 studies from around the world and concluded that "...at least a million animal and plant species - more than one in eight - already face extinction". This assessment is based on the idea of extinction debt (Hanski \& Ovaskainen, 2002), that is, the presumption that IUCN species listed as vulnerable (VU), endangered (EN) and critically endangered (CR) (accounting for $28 \%$ of the total species evaluated) will finally go extinct and, therefore, a similar percentage of the total biodiversity will also disappear. Notably, the majority of these endangered species live in the tropics, especially in the Indomalayan region, whereas North America and Australasia show smaller values (Fig. 3). According to the IPBES report, in which more than 130 countries participated, many of these extinctions could occur within decades because current extinction rates are "... at least tens to hundreds of times higher than the average over the past 10 million years". The main direct anthropogenic threats to biodiversity are (by order of importance) land/sea use change, direct exploitation, pollution, invasive alien species and climate change (IPBES, 2019). These are gross and imprecise estimates based on unwarranted assumptions and risky extrapolations, which do not favor public credibility. However, the need for urgent and radical economic, social and political changes is undeniable. Otherwise, major biodiversity losses will continue to 2050 and beyond.

\section{Conservation tips}

According to the above discussion, the current human-driven biodiversity crisis still does not qualify for a mass extinction in terms of the percentage of extinct species, but the current rates of biodiversity loss actually fit within the range of the five mass extinctions that occurred in the Phanerozoic. This should not be a criterion for deciding whether biodiversity conservation is a necessity, as this is indisputable, but is a serious warning about how quickly conservation actions should be taken. To discuss conservation strategies in detail is beyond the scope of this essay, but a brief comment on conservation targets seems pertinent. The magnitude and the high extinction rates of the current biodiversity crisis seem to have led to an obsession for conserving every living species. This not the idea. Species listed on the IUCN Red List as endangered should have priority, but conservation actions aiming to prevent other species from entering this category are also welcome. What is nonsense is to try to conserve every living species, which is contrary to the natural evolutionary process. If we were hypothetically able to preserve all species living on the planet, then we might prevent background extinction, thus stopping natural selection and, as a consequence, evolution. The world is as it is thanks to the speciation/extinction balance, which is fundamental for evolutionary diversification. It has been estimated that $99 \%$ of species that have lived on Earth, approximately $4 \cdot 10^{9}$ species, have gone extinct (Barnosky et al., 2011), most of them without 
human mediation. Stopping extinction is nonsensical in evolutionary terms and is as unnatural as favoring it.

\section{References}

Barnosky AD, Matzke N, Tomiya S, Wogan GOU, Swartz B, Quental TB, Marshall C, McGuire JI, Lindsey EL, Maguire KC et al (2011) Has the Earth's sixth mass extinction already arrived? Nature 471: 51-57.

Boehm, MAM, Cronk QCB (2021) Dark extinction: the problem of unknown historical extinctions. Biol Lett 17: 20210007.

Ceballos, G, Ehrlig PR, Barnosky AD, García A, Pringle RM, Palmer TM (2015) Accelerated modern human-induced species losses: entering the sixth mass extinction. Sci Adv 1: e1400253.

Hanski I, Ovaskainen O (2002) Extinction debt and extinction threshold. Cons Biol 16: 666-673.

Koch PL, Barnosky AD (2006) Late Quaternary extinctions: state of the debate. Annu Rev Ecol Evol Syst 37: 215-250.

IPBES (2019) Global assessment report of the Intergovernmental Science-Policy Platform on Biodiversity and Ecosystem Services. IPBES Secretariat, Bonn.

Lawton JH, May RM (1995) Extinction rates. Oxford University Press, Oxford.

Leakey R, Lewin R (1995) The Sixth Extinction. Patterns of Life and the Future of Humankind. Doubleday, New York.

Lomborg B (2001) The skeptical enviromentalist: measuring the real state of the world. Cambridge University Press, Cambridge.

Pimm SL, Russell GJ, Gittleman JL, Brooks TM (1995) The future of biodiversity. Science 269: 347-350.

Raup DM, Sepkosky JJ (1982) Mass extinctions in the marine fossil record. Science 215: 15011503.

Rull V (2021) Pristinity, degradation and replacement: the three dimensions of human impact on island vegetation. Prog Phys Geogr 45: 407-426.

Steadman DW (1995) Prehistoric extinctions of Pacific island birds: biodiversity meets zooarchaeology. Science 267: 1123-1131.

\section{Figure captions}

Figure 1. Taxonomic distribution of the 900 species that have gone extinct since 1500, according to the IUCN Red List of threatened species (https://www.iucnredlist.org/, retrieved 16 October 2021).

Figure 2. The five mass extinctions of the Phanerozoic, as defined by the percentage of extinct genera of marine animals. Each mass extinction is highlighted by a red number ( 1 to 5 ) and the percentage of extinct species with respect to the total. The orange line represents the background extinction rates (note that not all extinction bursts above background rates are considered mass extinctions). Geological periods: O, Ordovician; S, Silurian; D, Devonian; C, Carboniferous; P, Permian; Tr, Triassic; J, Jurassic; K, Cretaceous; Pg, Paleogene; N, Neogene. Based on Raup \& Sepkoski (1982).

Figure 3. World map of threatened species (vulnerable, endangered and critically endangered). Terrestrial species are in green, and marine species are in blue. The intensity of the colors is proportional to the number of endangered species. Modified from IPBES (2019). 


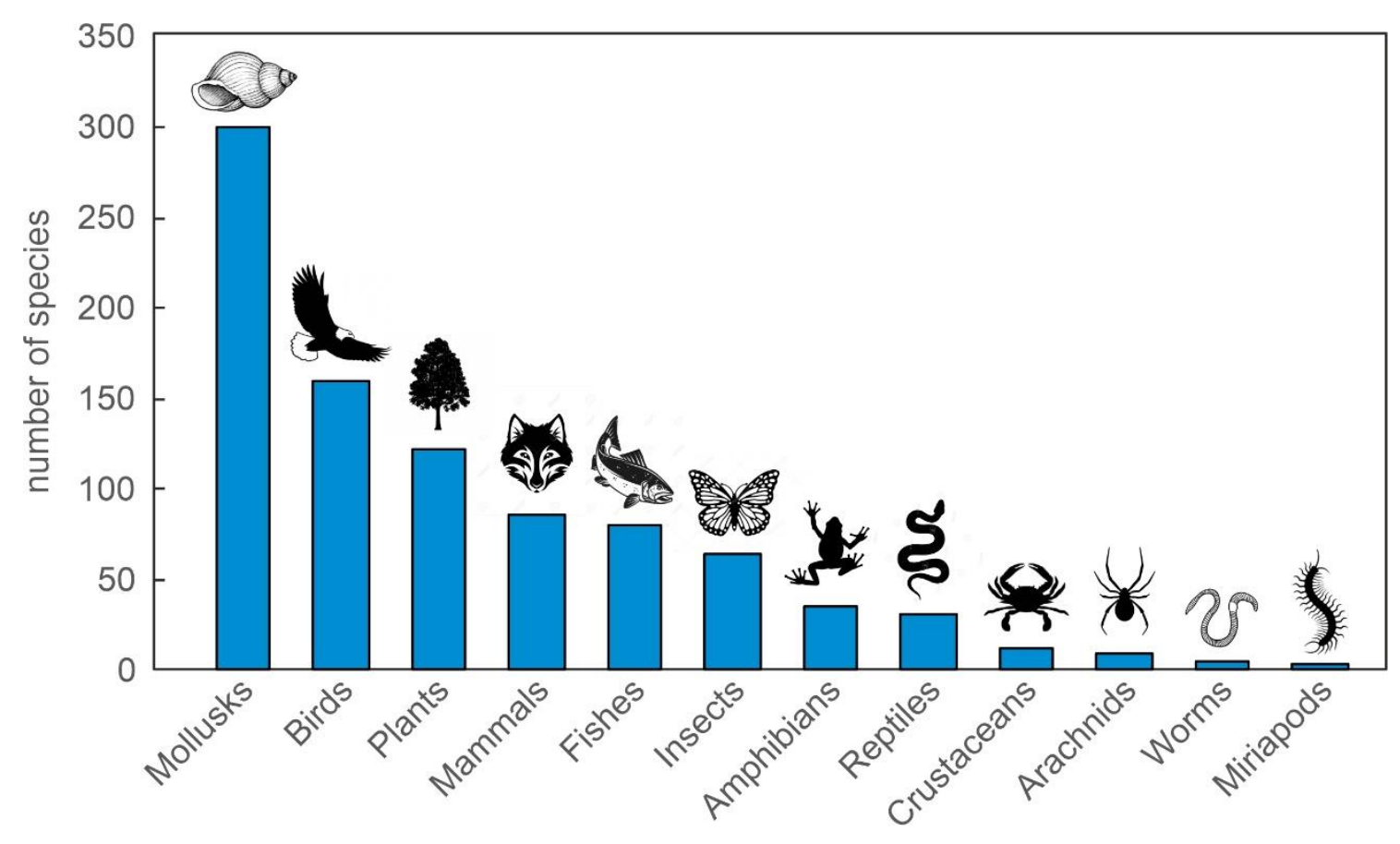

Figure 1

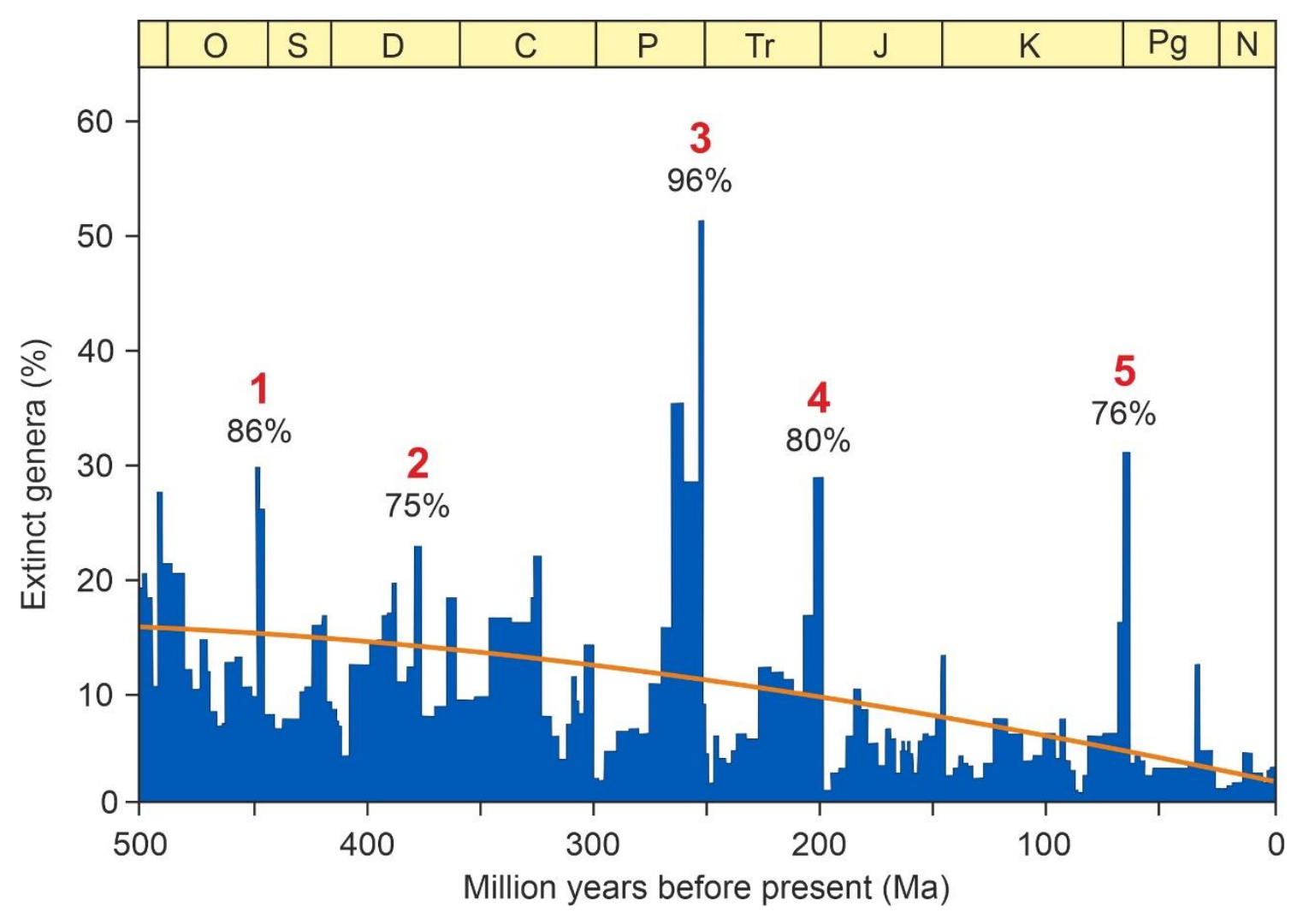

Figure 2 


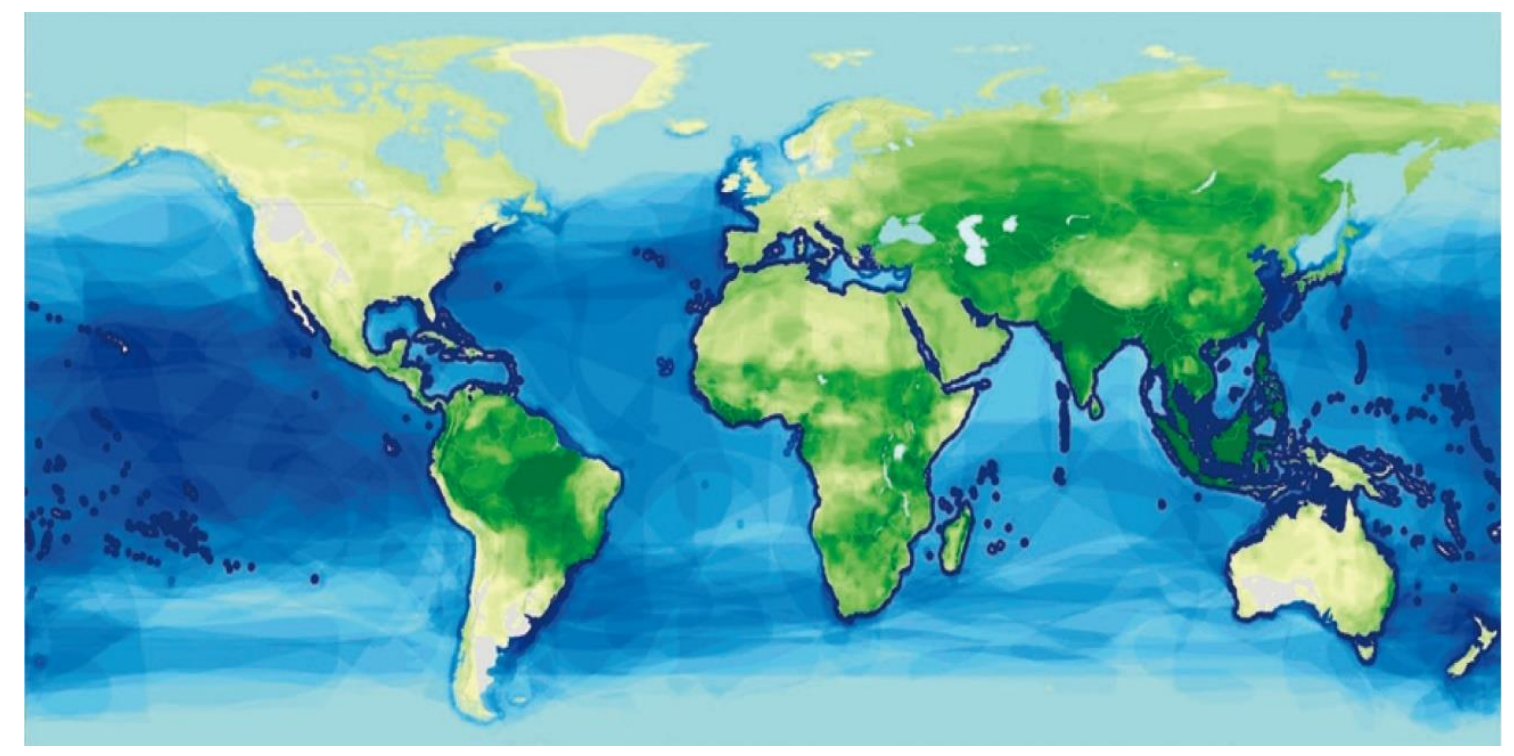

Figure 3 